\title{
Probing-Based Estimation of End-to-End Available Bandwidth
}

\author{
Seung Yeob Nam, Student Member, IEEE, Sunggon Kim, Junsu Kim, and Dan Keun Sung, Senior Member, IEEE
}

\begin{abstract}
A new mechanism to estimate available bandwidth for multiple hop routes is proposed based on a minimally backlogging concept. Since the proposed mechanism can estimate the available bandwidth quickly and track it adaptively, a reasonable range of available bandwidth for a short time interval can be obtained using the mean and variance of the estimated available bandwidth. The performance of the proposed mechanism is verified by simulation in a multiple hop network topology.
\end{abstract}

Index Terms-Available bandwidth, bandwidth estimation, probing, measurement, minimal backlogging.

\section{INTRODUCTION}

$\mathbf{I}$ T IS VERY important to monitor available bandwidth ( $\mathrm{AB})$ for efficient management of network resources and quality of service (QoS) guarantees of various types of internet flows. Several methods have been proposed to estimate this AB. Carter and Crovella [1] proposed a tool called the C-probe that can be used to estimate $\mathrm{AB}$ from the dispersion of trains of eight packets. They assumed that the dispersion of long packet trains is inversely proportional to AB. However, it was shown that this is not true by Dovrolis et al. [2]. Melander et al. [3] proposed a TOPP probing method that is an extension to the packet pair probing technique. However, TOPP is computationally intensive to implement. Recently, a tool called pathload was proposed by Jain and Dovrolis [4].

In this letter, we develop an $\mathrm{AB}$ estimation mechanism based on a simplified path model and a minimally backlogging concept. We focus on tracking the dynamically varying AB for a relatively short time period to finally obtain a reasonable range of the $\mathrm{AB}$. The performance of the proposed mechanism is verified by simulation in a multiple hop network topology.

\section{Minimally Backlogging Method}

Before considering the $\mathrm{AB}$ estimation problem for multiple hop routes, we introduce some fundamental concepts and theory for a single server. We consider a queueing system with a FirstCome-First-Served (FCFS) service policy. The service rate is $C$, and the arrival rate of packets except probing packets is $\lambda$. Let $L^{\prime}$ denote the average length of the packets. Then, for the queueing system, available bandwidth $C_{a}$ is defined as

$$
C_{a}=C(1-\rho)
$$

\footnotetext{
Manuscript received October 29, 2003. The associate editor coordinating the review of this letter and approving it for publication was Prof. I. Venieris.

The authors are with the Department of Electrical Engineering and Computer Science, Korea Advanced Institute of Science and Technology (KAIST), Daejeon, 305-701 Korea (e-mail: synam@cnr.kaist.ac.kr; sgkim@cnr.kaist.ac.kr; jskim@cnr.kaist.ac.kr; dksung@ee.kaist.ac.kr).

Digital Object Identifier 10.1109/LCOMM.2004.828180
}

where $\rho=\lambda L^{\prime} / C$. We now explain a probing scheme for a single server.

Definition 1: Suppose that we send probing packets into a queueing system so that there exists one and only one probing packet in the system. This probing method is called a minimally backlogging method.

If we send a probing packet into a queueing system just at the departure time of the previous probing packet, then there exists one and only one probing packet in the system. Let $\hat{Y}_{[s, t]}$ be the amount of probing packets served in a time interval $[s, t]$ when probing packets are sent to the queueing system according to the minimally backlogging method. Suppose that the size of probing packets is fixed to $L$. Then, we obtained in [5, Th. 7], that for a $G / G / 1$ queueing system

$$
\lim _{t \rightarrow \infty} E\left[\left|\frac{\hat{Y}_{[s, t]}}{t-s}-C(1-\rho)\right|^{q}\right]=0, \quad 0<q<\infty .
$$

The above equation implies that $L_{q}$ error converges to zero as the probing time duration goes to infinity. Thus, the service rate of minimally backlogging probing traffic can be used as an estimator of the AB.

\section{Network PATH Model}

For a path $\mathcal{P}$ consisting of $H$ serially connected links, AB $C_{a}$ for the path in a given time interval is defined as

$$
C_{a}=\min _{1 \leq i \leq H} C_{i}\left(1-u_{i}\right)
$$

where $C_{i}$ and $u_{i}$ denote the link rate and the utilization of the $i$ th link in the given time interval, respectively. The link with the least unused bandwidth of $C_{a}$ is referred to as tight link and the link with the minimum link rate is referred to as bottleneck link. We consider a single tight link because multiple tight links are not likely to occur frequently in real networks due to variation of the $\mathrm{AB}$ at each link. However, the proposed mechanism can be applied to multiple tight link environments. We consider the following simplified path model for a network path.

For a path consisting of multiple $(H \geq 1)$ hops, let $w_{h}, s_{h}$, and $g_{h}$ denote the waiting time, the service time, and the propagation delay of a packet at the $h$ th hop, respectively. Then, the end-to-end delay is $d=\sum_{h=1}^{H}\left(w_{h}+s_{h}+g_{h}\right)$. A tight link is assumed to occur at the $z$ th link. Let $d_{R}$ denote the summation of every $w_{h}$ and $s_{h}$ except those for the $z$ th link. If we let the expectation of $d_{R}$ be $\bar{d}_{R}$ and put $d_{R}-\bar{d}_{R}=\tilde{d}_{R}$, then the end-to-end delay can be expressed as

$$
d=\sum_{h=1}^{H} g_{h}+\bar{d}_{R}+\tilde{d}_{R}+\left[w_{z}+s_{z}\right]
$$

Since the propagation delay $g_{h}$ 's have fixed values and $\bar{d}_{R}$ is the expectation of $d_{R}$, the values of the first and second terms of (2) 


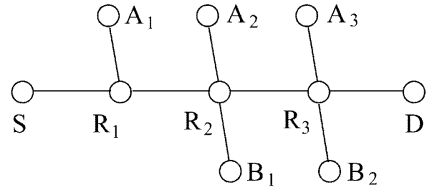

(a)

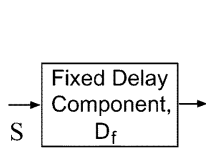

(b)
Fig. 1. An end-to-end network path model. (a) Network topology. (b) End-to-end path model.

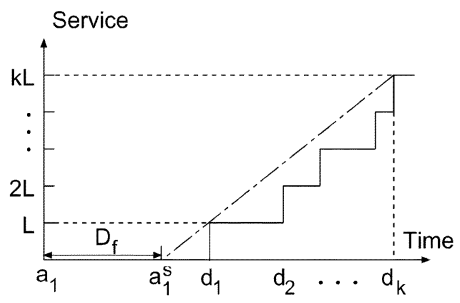

(a)

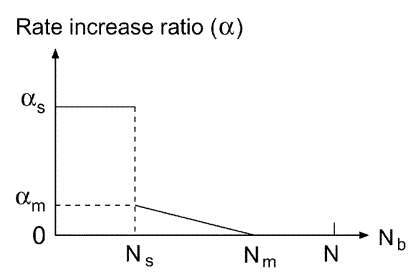

(b)
Fig. 2. The probing rate adaptation scheme. (a) Example of a service curve. (b) Rate increase ratio curve.

are constant and their sum is denoted as $D_{f} . E\left[\tilde{d}_{R}\right]=0$ and if we neglect the term of $\tilde{d}_{R}$, the remaining term is the queueing delay of $w_{z}+s_{z}$ at the tight link. Then, we can obtain a path model consisting of a fixed delay component $\left(D_{f}\right)$ and a virtual server $\mathcal{S}$ for the tight link as shown in Fig. 1.

Suppose that a probing packet $p$ arrives at the path at time $a_{p}$ and departs from the path at time $d_{p}$. Then, the packet $p$ arrives at the virtual server $\mathcal{S}$ at time $a_{p}^{s}=a_{p}+D_{f}$. When the packet arrives at the destination node, it departs from both the path and the virtual server $\mathcal{S}$. The virtual server is continuously backlogged for $k(k \geq 2)$ probing packet transmissions from the $j$ th probing packet in the interval $\left[a_{j}^{s}, d_{j+k-1}\right]$ if

$$
d_{j+m} \geq a_{j+m+1}^{s}, \quad \text { for all } 0 \leq m \leq k-2 .
$$

\section{Available Bandwidth Estimation Mechanism}

Sending minimally backlogging probing packets to the virtual server $\mathcal{S}$, we can estimate the $\mathrm{AB}$ for the path. We send a packet train of $N$ probing packets for a path and the time interval of $\left[a_{1}, d_{N}\right]$ is called a probing period. Then, $\mathrm{AB}$ for the path is estimated as follows.

If $N$ probing packets are sent according to the minimally backlogging method, then, by (1), $\mathrm{AB}$ for the virtual server in the interval $\left[a_{1}^{s}, d_{N}\right]$ can be estimated by $N L /\left(d_{N}-a_{1}^{s}\right)$, where $a_{1}^{s}=a_{1}+D_{f}$ and $D_{f}$ is the fixed delay for the current probing period. In real applications, several busy periods of probing packets may exist during a probing period. Consider the $i$ th busy period containing $k$ continuously backlogged probing packets ${ }^{1}$. Probing packets arriving during the busy period are indexed from 1 to $k$. Fig. 2(a) illustrates a sample service curve for the busy period showing the amount of probing packets served for $\left[a_{1}, t\right]$. The Measured Probing Rate (MPR) for the $i$ th busy period is defined as

$$
\operatorname{MPR}(i)=\frac{k L}{d_{k}-a_{1}^{s}}=\frac{k L}{\left(d_{k}-a_{1}\right)-D_{f}} .
$$

${ }^{1}$ When we check the continuous backlogging condition of (3) for the current probing period, the $D_{f}$ value calculated in the previous probing period is used.
The $M P R$ for the longest busy period during a probing period is used to reliably estimate $\mathrm{AB}$.

We use the value of $D_{f}$ calculated in the previous probing period to estimate the value of $D_{f}$ for the current probing period. If we assume that the service rate for the first packet and the average service rate for the other $k-1$ packets are the same, then we can estimate $D_{f}$ as

$$
\tilde{D}_{f}=\left(d_{1}-a_{1}\right)-\frac{d_{k}-d_{1}}{k-1} .
$$

$D_{f}$ is estimated at the longest busy period while satisfying $a_{p}^{s} \leq$ $d_{p}$ for any probing packet $p$.

Let $N_{b}=\max _{i} N_{b}(i)$, where $N_{b}(i)$ is the number of probing packets belonging to the $i$ th busy period in a probing period. We try to maintain $N_{b}$ within a reasonable range by an adaptive probing scheme. A small value of $N_{b}$ is due to a lower probing rate than for minimal backlogging and a large value of $N_{b}$ is due to a higher rate. If $N_{b}$ is in the reasonable range, we may assume that the minimal backlogging occurs. Thus, $M P R$ is a reliable estimate of the AB. Let $\left(N_{s}, N_{m}\right]$ be the reasonable range of $N_{b}$. Fig. 2(b) shows the proposed probing rate adaptation scheme, which is explained as follows.

Case 1) If $N_{b}>N_{m}$, then $M P R$ is considered to be larger than the $\mathrm{AB}$ due to a higher probing rate than for minimal backlogging, and the next input rate is set to $M P R$. The $\mathrm{AB}$ is estimated by $M P R$ since $M P R$ quickly approaches to the AB.

To give a reason for the use of $M P R$ as the next probing rate, we consider an example. For an FCFS server with a link rate of $C$ and an $\mathrm{AB}$ of $C_{a}$, if the probing packets arrive at a rate of $r\left(\geq C_{a}\right)$, they are served at a rate of

$$
m(r)=\frac{r}{C-C_{a}+r} C .
$$

If we adjust $(n+1)$ th probing rate by $r_{n+1}=m\left(r_{n}\right)$, then it can be easily shown that $\lim _{n \rightarrow \infty} r_{n}=C_{a}$.

Case 2) If $N_{b} \leq N_{s}, M P R$ for this short busy period may be inaccurate because the minimal backlogging condition is not satisfied. Thus, the current $A B$ is estimated by the $\mathrm{AB}$ at the last probing period for $N_{b}>N_{s}$. If $N_{b} \leq N_{s}$ consecutively $i$ times since the last probing period with $N_{b}>N_{s}$, then the next input rate is set to $\mathrm{AB} \cdot\left(1+\alpha_{s}\right)^{i}$.

Case 3) If $N_{s}<N_{b} \leq N_{m}$, then $M P R$ is a reliable estimate of the AB. However, it is necessary to maintain the probing rate slightly higher than $\mathrm{AB}$ in order to obtain a reliable value of $M P R$. Thus, the next input rate is increased to $M P R \cdot\left(1+\alpha\left(N_{b}\right)\right)$, where $\alpha\left(N_{b}\right)=\alpha_{m}\left(N_{m}-N_{b}\right) /\left(N_{m}-N_{s}\right)$, and $\alpha_{m}$ is the maximum rate increase ratio in the medium busy period range.

\section{NumericAl RESUlts}

We compare the performance of the proposed $\mathrm{AB}$ estimation mechanism with that of pathload [4] through OPNET simulation. A multiple hop topology is illustrated in Fig. 1(a). Each node is modeled as an output queued router with a FIFO queue. We estimate $\mathrm{AB}$ for the path $S-R_{1}-R_{2}-R_{3}-D$. Every 


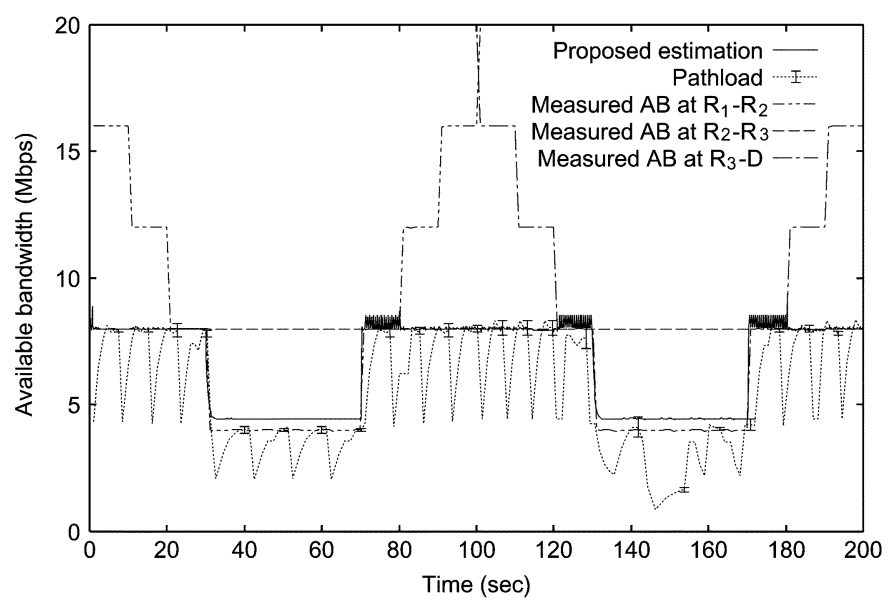

Fig. 3. Comparison of available bandwidths estimated by the proposed mechanism and by the pathload under a CBR traffic load.

link except $R_{2}-R_{3}$ has a link rate of $20 \mathrm{Mb} / \mathrm{s}$ and a propagation delay of $5 \mathrm{~ms}$. Link $R_{2}-R_{3}$ with a link rate of $10 \mathrm{Mb} / \mathrm{s}$ is the bottleneck link. The sizes of both probing packets and data packets are 4000 bits. For the proposed mechanism, the number of probing packets sent in one probing period $(N)$ is 100 . Since the proposed probing scheme attempts to send probing packets at a slightly higher rate than the $\mathrm{AB}$, the load offered to the tight link may exceed 1 a little during a probing period. In order to prevent overload for the tight link, consecutive probing periods are separated by at least one probing period length of $d_{N}-a_{1}$. The values of the rate adaptation related parameters are set to $N_{m}=0.95 \times N=95, N_{s}=0.30 \times N=30, \alpha_{m}=0.10$, and $\alpha_{s}=1.0$. For the pathload [4], the user-specified resolution of $\mathrm{AB} \omega$ is set to $0.2 \mathrm{Mb} / \mathrm{s}$ and the grey-region resolution $\chi$ is $0.3 \mathrm{Mb} / \mathrm{s}$.

Two types of traffic patterns are used for nonprobing packet sequence: constant bit rate (CBR) and self-similar traffic. Selfsimilar traffic is generated using a multi-fractal model [6]. The Hurst parameter is 0.8 and the sigma/mean ratio of a flow is approximately 0.5 . The mean rate of each flow is $4 \mathrm{Mb} / \mathrm{s}$ except a flow which is sent from $A_{2}$ to $B_{2}$ and has a rate of $2 \mathrm{Mb} / \mathrm{s}$. During a simulation time of 200 seconds, 4 flows with a lifetime of 70 seconds are sent on route $A_{1}-R_{1}-R_{2}-B_{1}$ sequentially at an interval of 10 seconds from time 0.4 flows with a lifetime of 70 seconds are sent on route $A_{3}-R_{3}-D$ sequentially at an interval of 10 seconds from time 100 . Thus, link $R_{1}-R_{2}$ is a tight link in the interval $[20,80]$. Link $R_{2}-R_{3}$ is a tight link in the intervals of $[0,30],[70,130]$, and $[170,200]$. Link $R_{3}-D$ is a tight link in the interval $[120,180]$. Thus, two tight links exist in the intervals of $[20,30],[70,80],[120,130]$, and [170, 180].

Fig. 3 compares the $\mathrm{AB}$ of the proposed mechanism with that of the pathload under a CBR traffic load. The pathload iteratively estimates the range $\left[R^{\mathrm{min}}, R^{\max }\right]$ of $\mathrm{AB}$. The trace of $\left(R^{\min }+R^{\max }\right) / 2$ is plotted in Fig. 3 and the range of $\left[R^{\min }, R^{\max }\right]$ is also shown at the instant of termination. The pathload is restarted just after it terminates. We can observe that it takes about 8 seconds for the pathload to terminate. The pathload sometimes yields a significant error in the estimation of $\mathrm{AB}$, especially at time 153.8 as shown in Fig. 3. However, the

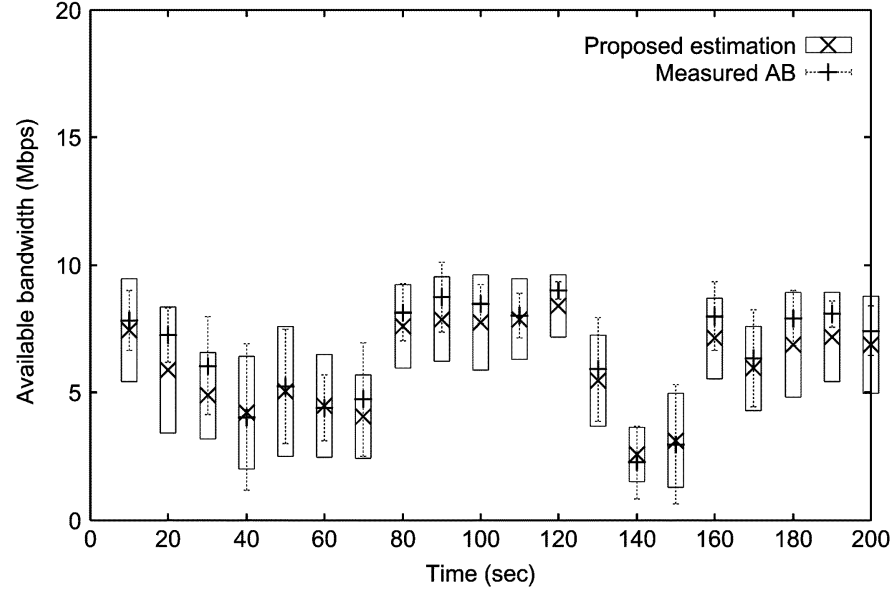

Fig. 4. Available bandwidth estimated by the proposed mechanism under a self-similar traffic load.

proposed mechanism closely tracks the $\mathrm{AB}$ even if $\mathrm{AB}$ changes abruptly or there exist two tight links. The error observed in the intervals of $[30,70]$ and $[130,170]$ is due to the fact that the proposed probing scheme tries to maintain the probing rate slightly higher than the $\mathrm{AB}$ to obtain a reliable value of $M P R$. If $N_{m}$ or $\alpha_{m}$ is decreased, this error can also be decreased, but more ripples may occur due to unreliable values of MPR.

Fig. 4 compares the mean $(\mu)$ and the standard deviation $(\sigma)$ of the $\mathrm{AB}$ estimated by the proposed mechanism with those of the measured $\mathrm{AB}$ under a self-similar traffic load. The range of $[\mu-\sigma, \mu+\sigma]$ is plotted based on the measurement at an interval of 10 seconds. It is difficult to obtain reasonable mean and sigma of $\mathrm{AB}$ for a short period of 10 seconds by the pathload because of a long convergence time. We can observe that the mean of the measured AB lies within $\sigma$ from $\mu$ of the estimated $\mathrm{AB}$ for every estimation time.

\section{CONCLUSION}

A new $\mathrm{AB}$ estimation mechanism for a specific path is proposed based on the minimally backlogging concept and a simplified path model. Since the proposed mechanism tracks the $\mathrm{AB}$ rather accurately even when the $\mathrm{AB}$ changes abruptly, it can be used to obtain a reasonable range of dynamic $\mathrm{AB}$ in a short time interval.

\section{REFERENCES}

[1] R. L. Carter and M. E. Crovella, "Measuring bottleneck link speed in packet-switched networks," Perform. Eval., vol. 27-28, pp. 297-318, 1996.

[2] C. Dovrolis, P. Ramanathan, and D. Moore, "What do packet dispersion techniques measure?," in Proc. IEEE Infocom'01, Apr. 2001, pp. 905-914.

[3] B. Melander, M. Bjorkman, and P. Gunningberg, "A new end-to-end probing and analysis method for estimating bandwidth bottlenecks," in Proc. IEEE Globecom'00, Nov. 2000, pp. 415-421.

[4] M. Jain and C. Dovrolis, "Pathload: A measurement tool for end-to-end available bandwidth," in Proc. Passive and Active Measurements (PAM) Workshop, Mar. 2002, pp. 14-25.

[5] S. Y. Nam, S. Kim, and D. K. Sung, "Estimation of available bandwidth for an unidentified queueing system," IEEE Trans. Commun., submitted for publication.

[6] R. H. Riedi, M. S. Course, V. J. Ribeiro, and R. G. Baranuik, "A multifractal wavelet model with application to network traffic," IEEE Trans. Inform. Theory, vol. 45, pp. 992-1018, Apr. 1999. 